\title{
Variable UV Line Emission in S Carinae Miras Do Not Fear Change
}

\author{
E.W. Brugel ${ }^{1}$, R. Davis ${ }^{1}$, J. Bookbinder ${ }^{2}$ \\ ${ }^{1}$ CASA, University of Colorado, Boulder, CO, ${ }^{2} S A O$, Cambridge, MA
}

\begin{abstract}
The object $\mathrm{S}$ Carinae is a bright, relatively hot, short $\left(149^{d}\right)$ period mira. As expected, the $\mathrm{Mg}$ II $h$ and $k$ lines dominate the ultraviolet line spectra during a large fraction of the pulsation cycle. There are also numerous emission lines of Fe I and Fe II produced by either fluorescense or possibly collisional excitation. The unifying "theme" for all the ultraviolet line emission is variability - during a pulsation cycle one observes changes in the intensity, the central wavelength and (for the stronger lines) the line profile.
\end{abstract}

During the period of $11 / 87$ - 5/91 we obtained 23 high dispersion long wavelength IUE images of $S$ Car. The majority of the data are concentrated during three distinct pulsation cycles. Cycle 1: Seven spectra were taken during a 44 day interval covering phases 0.23 to 0.53 . Cycle 2: Six spectra were taken during a 53 day interval covering phases 0.05 to 0.40 . Cycle 3: Ten spectra were taken during a 62 day interval covering phases 0.40 to 0.82 . Nine of the spectra were taken during a 4 day period, covering phases 0.40 to 0.43 .

A general description of these observations follows:

1. Optical Light Curve: The optical (i.e. Fes) light curve is fairly constant from cycle to cycle, though the ultraviolet line fluxes change significantly from one cycle to the next.

\section{Mg II Emission Lines:}

(a) The Mg II flux peaks near phase 0.25.

(b) Both the Mg II $h$ and $k$ lines are confined to the blueward side of line center in the reference frame of the star.

(c) The observed ratio of the $\mathrm{Mg}$ II $k$ to $h$ line fluxes is always $<0.6$. The theoretically expected $k / h$ ratio should lie between two (for an optically thick atmosphere) and unity (for an optically thin atmosphere) (Bookbinder, et al. 1989). Recent models by Luttermoser (private commun.) indicate that "mutilation" of the Mg II $k$ line may be due to radiative transfer effects. 
3. Fe I and Fe II Emission Lines: In addition to Mg II, there are approximately a hundred unique emission lines detected at the three $\sigma$-level. Some lines are detected only once, however the majority are seen on 4 to 25 different spectra. A few of the lines with multiple detections are discussed. Variations in line intensity, velocity (or more precisely, the line centroid) and profiles are seen.

(a) Fe II UV $1(2625.669 \AA)$ line: This is one of the strongest Fe II lines and is seen at almost all phases (it has also been detected in other miras). The cycle 2 data show a moderate variation in intensity, with the peak flux occurring at approxiamtely the same phase as the peak in $\mathrm{Mg}$ II. Cycle 3 data show approximately constant flux over the small phase interval 0.40 0.43 , and no detectable flux at phase 0.82 . There is however, a significant change in the central wavelength during the cycle. A modest red-shift occurs from phase 0.40 to 0.41 to 0.42 , then a dramatic blue-shift of $0.4 \AA$ (or $\approx 45 \mathrm{~km} / \mathrm{s}$ ) occurs from phase 0.42 to 0.43 .

(b) Fe II UV $32(2732.446 \AA, 2759.332 \AA$, and $2775.338 \AA)$ lines: As expected, since these line are all from the same multiplet, their behavior is fairly consistent. The cycle 2 data show a continuous increase in flux from phase 0.05 to 0.40 - though the $2775 \AA$ line deviates from the pattern. Cycle 3 data show approximately constant flux over the small phase interval 0.40 0.43 , and no detectable flux at phase 0.82 . Similar to Fe II UV 1 , there is a significant change in central wavelength during the cycle. A modest red-shift occurs from phase 0.40 to 0.41 to 0.42 , then a dramatic blue-shift of $0.4 \AA$ (or $\approx 45 \mathrm{~km} / \mathrm{s}$ ) occurs from phase 0.42 to 0.43 .

(c) Fe I UV $44(2823.276 \AA)$ line: This line is produced by fluorescence via pumping from the Mg II $k$-line (Carpenter et al. 1988).

\section{References:}

Bookbinder, J., Brugel, E.W. and Brown, A., 1989 Astroph. J., 342, 516.

Carpenter, K.G., Pesce, J.E., Stencel, J.E., Brown, A., and Wing, R.F. 1988 Astroph. J. Supp., 68, 345.

\section{Acknowledgements:}

This research was funded by NASA grant NAG5-350 to the University of Colorado and NASA grant NAG5-87 to the Smithsonian Astrophysics Observatory. 